\title{
Fossil Magnetospheres Confront Newborn Dynamos in the Rapid Braking Zone
}

\author{
Thomas R. Ayres \\ Center for Astrophysics $\&$ Space Astronomy, University of Colorado, \\ Boulder, CO, USA, 80309-0389
}

\begin{abstract}
Fast spinning Hertzsprung gap giants display super-rotational broadening of UV "hot" lines like Fe XXI $\lambda 1354$ and C IV $\lambda 1548$, with FWHM's up to twice that expected from the photospheric $v \sin i$. This possibly is the result of extended fossil magnetospheres enveloping the gap giants, a new type of stellar corona. The magnetospheric phase is short-lived, however, as the rapidly evolving giants develop a competing dynamo-generated surface field in the so-called Rapid Braking Zone.
\end{abstract}

\section{Introduction}

The hot $\left(T \sim 10^{6}-10^{7} \mathrm{~K}\right)$ coronae of late-type stars are thought to be intimately connected to stellar rotation through the spin-catalyzed magneto-convective "dynamo" (Rosner \& Vaiana 1978). Rotation not only is an underlying physical cause of coronal activity, but also offers a way to probe the magnetically disturbed areas on the stellar surface through observational techniques such as starspot photometry and Doppler imaging (Vogt \& Penrod 1986). What's more, a high level of magnetic activity in a fast-spinning star undoubtedly powers a strong coronal wind, which in turn removes angular momentum, thereby braking the star's rotation. The negative feedback ultimately quenches coronal activity in solar-mass single stars, acting most strongly over the first $\sim 100$ million years of the Main sequence lifetime (Stauffer 1991). The braking episode is even more dramatic among moderate-mass $\left(\sim 3 \mathrm{M}_{\odot}\right)$ giants in the Hertzsprung gap, during the short-lived first crossing. In what might be called the "Rapid Braking Zone" (RBZ), rotation rates of moderate-mass giants fall precipitously from ¿ $50 \mathrm{~km} \mathrm{~s}^{-1}$ to only a few $\mathrm{km} \mathrm{s}^{-1}$ over just a few spectral classes $(\mathrm{G} 0 \rightarrow \mathrm{G} 5$; Gray 1981), in the course of perhaps only a million years.

I, and my colleagues, have proposed (Ayres et al. 1998) that such stars initially arrive at the blue edge of the gap with a fossil magnetosphere surviving from the pre-MS phase (left side, Fig. 1). The large-scale dominantly dipolar field becomes activated by weak surface convection when the evolving giant reaches the early-F spectral types, giving rise to an extended hot corona (middle, Fig. 1). The surface convection strengthens as the star continues to evolve redward, eventually becoming strong enough to shred the roots of the fossil magnetosphere, and instigate a more solar-like dynamo. That, coupled with the fast rotation inherited from the MS progenitor, produces an episode of strongly enhanced coronal activity (right side, Fig. 1). But, with the enveloping "dead zone" of the magnetosphere eliminated, the coronal wind would be free to carry 


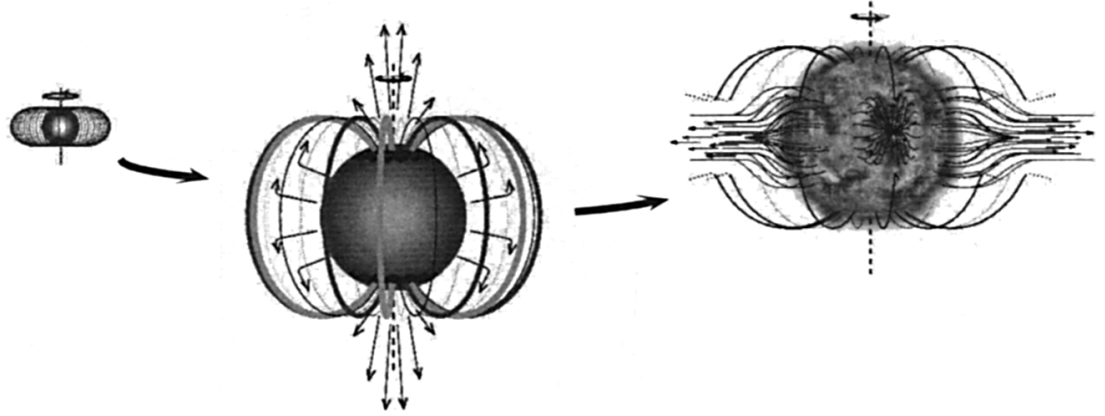

Figure 1. Coronal evolutionary scenario for $\mathrm{a} \sim 3 \mathrm{M}_{\odot}$ star (Ayres et al. 1998)

away angular momentum - a substantial amount, boosted by the large Alfvén radius of the giant - thereby quickly braking the stellar rotation, and ending the brief period of coronal hyperactivity.

Figure 2 sets the context of the transition between the proposed "magnetospheric" Hertzsprung gap giants and those in the RBZ which are developing more solar-like, albeit quite active, dynamos. The diagram compares coronal $\mathrm{X}$-rays (as measured by ROSAT) against the subcoronal tracer $\mathrm{C}$ IV. The different zones are - (1) F8-K5 dwarfs and G2-7 giants, (2) F0-G1 Hertzsprung gap giants, (3) short-period RS CVn binaries, (4) active clump (G8-K0) giants, (5) low-activity clump giants, (6) early-G "hybrid" supergiants, and (7) "noncoronal" red giants (K1-M III). A distinguishing characteristic of the gap giants is that they are "X-ray deficient" compared to G/K dwarf stars and active cooler giants (Simon \& Drake 1989). Several interesting objects are marked in the diagram: "FK" = FK Com HD 117555 (G2 III; $v_{\text {rot }} \sim 160 \mathrm{~km} \mathrm{~s}^{-1}$ ), an ultra-fast spinning giant (Bopp \& Stencel 1981) probably in the early RBZ phase; "31" = 31 Com HD 111812 (G0 III) and "Ab" $=\alpha$ Aur Ab HD 34029b (Capella G1; G1 III) are classical gap giants with $v_{\text {rot }} \sim 50 \mathrm{~km} \mathrm{~s}^{-1}$. "HR" $=$ HR $9024 \mathrm{HD}$ 223460 (G1 III) and "24" = 24 UMa HD 82210 (G4 III) are more slowly spinning post-gap giants, probably in the middle of the RBZ dynamo-braking phase. " $\mu "=\mu$ Vel HD 93497 (G5 III) is even further along in the braking process, and is comparably active to the most extreme of the post helium flash "clump" giants (like $\beta$ Cet [K0 III] HD 4128 ).

\section{New Observations}

The Far-Ultraviolet Spectroscopic Explorer (FUSE; Moos et al. 2000) and the Hubble Space Telescope Imaging Spectrograph (HST STIS; Woodgate et al. 1998) are revolutionizing the study of the hot coronal outer atmospheres of late-type stars through access to coronal forbidden lines such as Fe XVIII $\lambda 974$ $\left(10^{6.8} \mathrm{~K}\right)$ in the FUSE band and Fe XXI $\lambda 1354\left(10^{7.0} \mathrm{~K}\right)$ available to STIS. Although these features generally are faint, the high sensitivities of FUSE and 


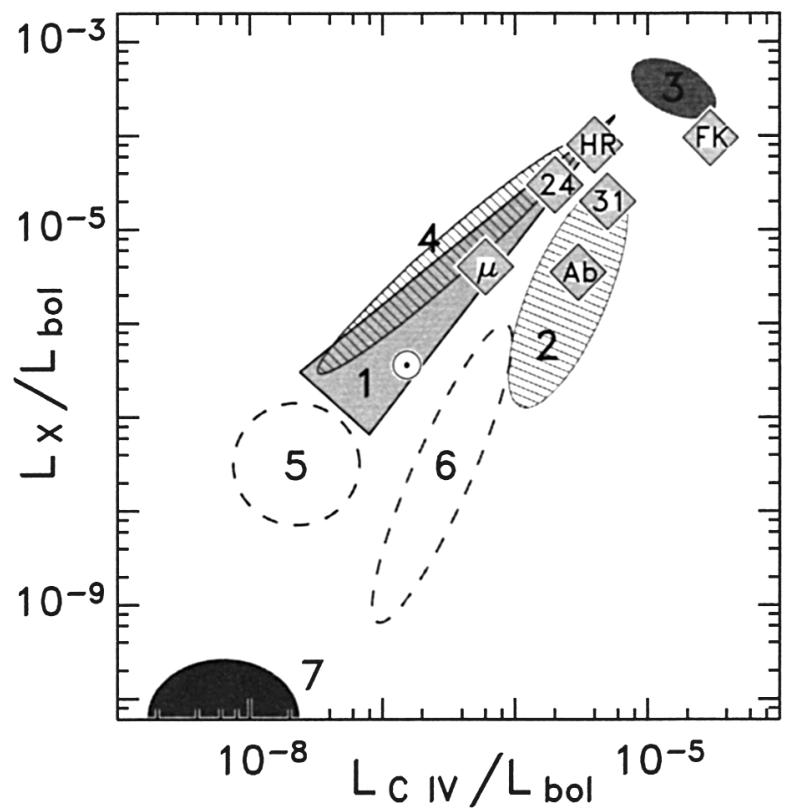

Figure 2. "Flux-flux" diagram: X-rays $\left(T>10^{6} \mathrm{~K}\right)$ vs. C IV $\left(10^{5} \mathrm{~K}\right)$.

especially $H S T$ have permitted useful observations in a dozen or so coronally active bright stars (Ayres et al. 2003; Redfield et al. 2003). The primary motivation for going after the coronal forbidden lines is that the FUSE and $H S T$ spectrographs can fully resolve the thermal widths of the features, whereas even the powerful High Energy Transmission Grating Spectrometer (HETGS) on the Chandra X-ray Observatory falls short in resolution by a factor of about 10 in its observations of coronal resonance lines in the $1 \mathrm{keV}$ "iron L-shell." The UV high-temperature forbidden lines potentially can help stellar coronal physicists achieve the "holy grail" of recording the crucial dynamical part of the energetics of flare events, but the hot Fe features already have provided provocative information on the extended coronal outer atmospheres of fast-spinning stars, through the phenomenon of "super-rotational broadening," previously identified in $10^{5} \mathrm{~K}$ subcoronal emissions such as $\mathrm{C}$ IV $\lambda 1548$ (Ayres et al. 1998).

Figure 3 compares HST STIS spectra of chromospheric $\mathrm{Cl}$ I, subcoronal $\mathrm{O} \mathrm{V}$, and coronal Fe XXI in two fast-rotating Hertzsprung gap giants: $31 \mathrm{Com}$ HD 111812 and Capella G1 HD 34029b (see Fig. 2, above). Points and error bars (where visible) represent the observed profiles, corrected for blends and low-amplitude "broad components" (see Wood, Linsky, \& Ayres 1997). Notea different flux scaling applies to each panel: $\mathrm{Cl}$ I $\lambda 1351$ (which is radiatively pumped by $\mathrm{C}$ II $\lambda 1335$ ) usually is $\sim 5 \times$ brighter than the $\mathrm{O} \mathrm{V}$ line, which in turn typically is $\lesssim 2 \times$ brighter than Fe XXI. Solid curves are the best-fit rotational profiles, which are "spun-up" versions of reference lineshapes, according to the $v \sin i$ values (in $\mathrm{km} \mathrm{s}^{-1}$ ) listed in the upper left corner of each panel. The reference profiles are the thin dot-dashed curves - scaled down for clarity-taken 


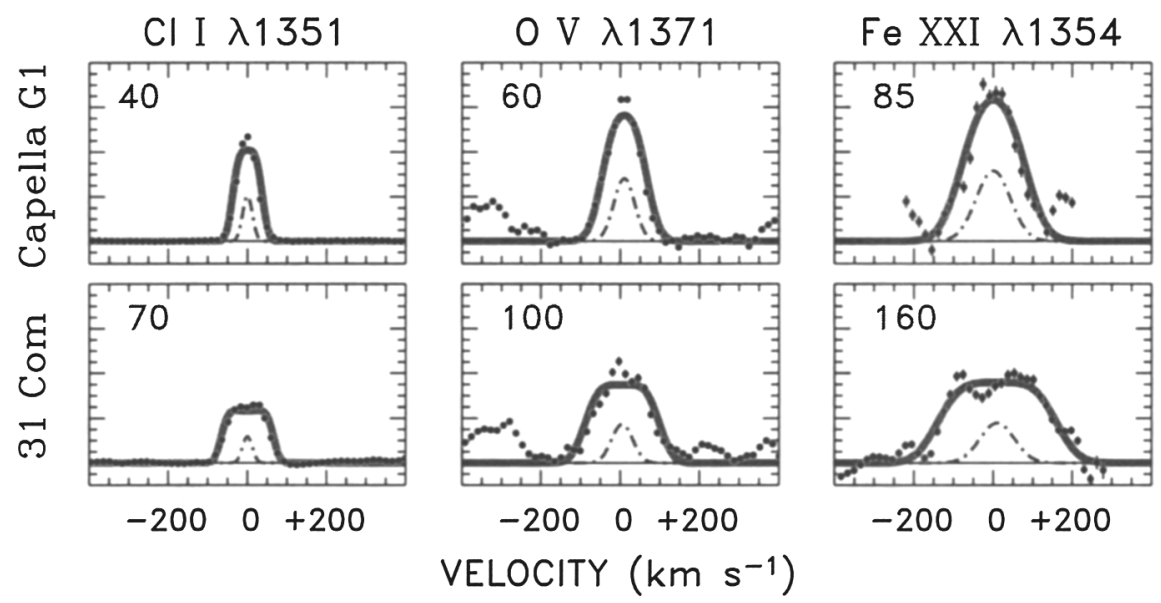

Figure 3. HST STIS spectra of chromospheric $\mathrm{Cl}$ I, subcoronal $\mathrm{O} \mathrm{V}$, and coronal Fe XXI in two fast-rotating Hertzsprung gap giants.

from the slowly rotating active clump giant $\beta$ Cet HD 4128 (K0 III). The fitted $\mathrm{Cl}$ I widths are compatible with $v \sin i$ 's from visible spectra. The higher temperature features, however, show evidence of excess broadening, which appears to increase with increasing formation temperature. (The $\mathrm{O} V$ intersystem line is fainter than permitted lines that arise near its $2 \times 10^{5} \mathrm{~K}$ formation temperature, but it has the important advantage in $v \sin i$ studies of being optically thin, and therefore not susceptible to "opacity broadening.")

Figure 4 illustrates average emission line profiles from representative giant stars observed by STIS in the 1150-1700 $\AA$ region. The larger profiles represent a superposition of the Si IV $1400 \AA$, C IV $1550 \AA$, and N V $1240 \AA$ doublets, which form over overlapping temperature ranges $6 \times 10^{4}-2 \times 10^{5} \mathrm{~K}$ in the subcoronal "transition zone." Each of the six lines was scaled by its integrated flux and averaged with the others, filtering out deviant points. (The darker shaded envelopes indicate the rms of the average, which becomes large if one of the profiles locally is much different than the others, say due to an extraneous blend.) The small, darker profiles in each panel represent a similar average for a variety of low-excitation narrow chromospheric features, including $\mathrm{Cl}$ I $\lambda 1351$ and several bright lines of O I, C I, and S I. The average "hot-line" profiles are conspicuously broader than the low-excitation lineshapes, presumably owing to enhanced small-scale turbulence resulting from the excess mechanical heat deposition required to sustain the elevated temperatures of the subcoronal layers. In this regard, it is somewhat surprising that the six high-excitation lines in each star have such similar profiles (as indicated by the small rms), suggesting that the kinematic environment-although clearly enhanced over that of the chromosphere - does not change much over the broad temperature range covered by the three doublets.

The three stars in the top row all are fast rotators, as evidenced by the broad low-excitation average profiles. The star in the lower left hand corner, 


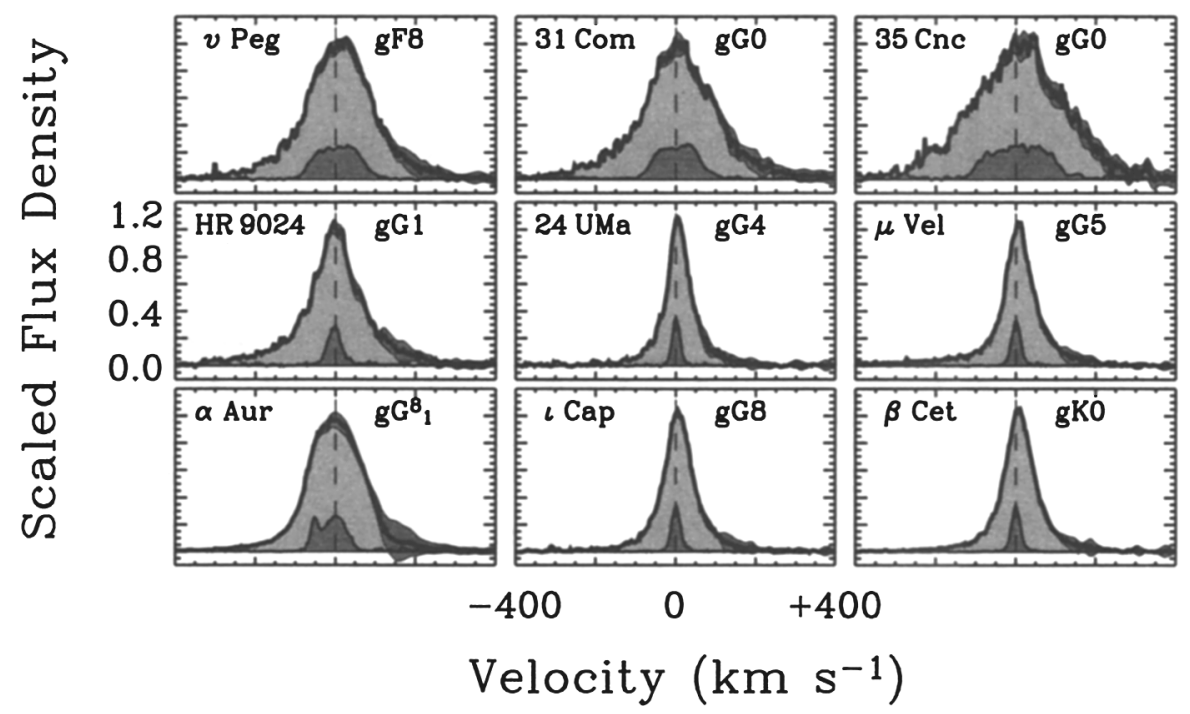

Figure 4. Average STIS hot-line and chromospheric profiles of F-K giants.

$\alpha$ Aur HD 34029 (G8 III + G1 III), is dominated in the UV hot lines by the G1 III secondary ("Ab"), a fast rotating Hertzsprung gap giant (Ayres \& Linsky 1980). A weak, sharp contribution due to the G8 III primary can be seen in the average low-excitation lineshape, Doppler shifted to the blue owing to the relative orbital motion at the time of observation. HR 9024 HD 223460 is a moderately fast rotator ( $v \sin i \sim 20 \mathrm{~km} \mathrm{~s}^{-1}$ ); which, like FK Com, appears to be in the early dynamo-braking phase.

\section{Discussion}

Figure 5 summarizes the apparent excess broadening seen in a variety of late$\mathrm{F}-\mathrm{K} 0$ giants, based on STIS average hot-line and chromospheric profile measurements (filled and open circles, respectively), like those depicted in Fig. 4. The dashed curves indicate expected line widths if the $v \sin i<6 \mathrm{~km} \mathrm{~s}^{-1}$ profiles are "spun up" according to the $v \sin i$ on the abscissa. The different curves for the hot-line case represent "super-rotational" broadening according to the radius enhancement factors indicated in the figure: $r \equiv R_{\mathrm{em}} / R_{\star}$, where $R_{\mathrm{em}}$ is the actual radius out to which the hot-line emission extends. Normal photospheric broadening is $r \equiv 1$, which also is anticipated for the hot lines if the subcoronal emission regions lie close to the stellar surface. In several cases illustrated, however, the hot line zones apparently extend out to $r \sim 2$, which corresponds to about ten scale heights above the altitude where $10^{5} \mathrm{~K}$ gas ordinarily would be found in hydrostatic equilibrium. These new STIS observations reinforce the picture developed previously - with spectrally much more restricted GHRS observations (Ayres et al. 1998) - that the $10^{5} \mathrm{~K}$ emission regions of the 


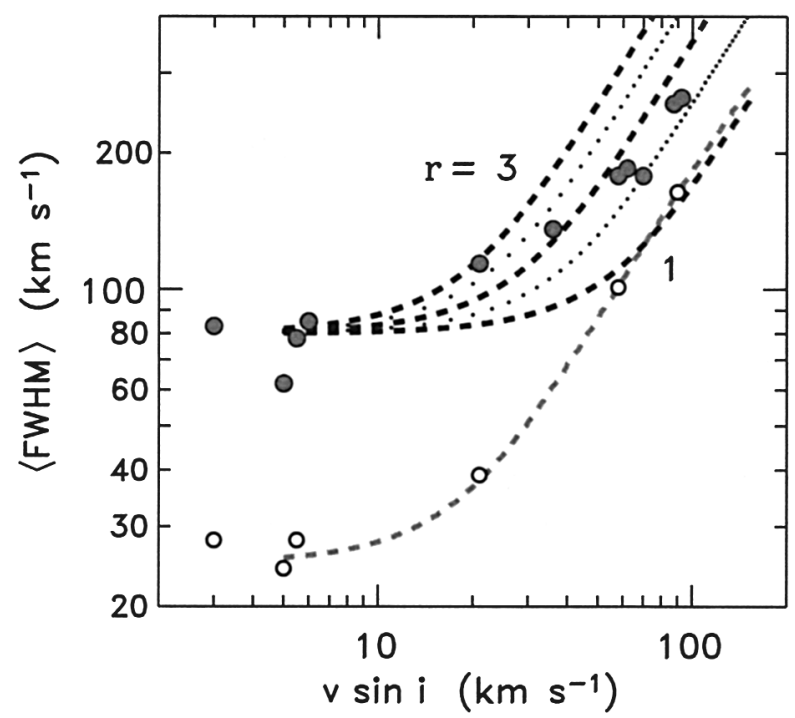

Figure 5. Rotational broadening measured in hot and cool UV lines of $\mathrm{F}-\mathrm{K}$ giants.

fast-rotating Hertzsprung gap giants are significantly extended in altitude, in line with the magnetospheric scenario proposed in the earlier work. Furthermore, the STIS observations of Fe XXI (and companion FUSE measurements of Fe XVIII) indicate that the hot coronal regions also are highly extended, perhaps even more so than the subcoronal zones.

In the final analysis, stellar rotation not only is a key player in the dynamo mechanism underlying stellar magnetic activity, but also is a valuable observational tool that allows us to image surface structure on active stars, and probe the geometry of their extended coronal zones, simply through high dispersion UV spectroscopy; avoiding the extremely challenging milliarcsecond resolution otherwise necessary in a direct imaging experiment. The coronal spatial resolution achieved by super-rotational broadening is especially valuable for addressing the apparently profound changes that occur in the evolution of coronal magnetic structure through the region of the Rapid Braking Zone. Some preliminary results have been described here, but clearly a great deal of work remains to be done.

Acknowledgments. This study was supported by several HST and FUSE Guest Observer grants to the University of Colorado. NASA/ESA HST, observations were collected at the STScI, operated by AURA, under contract NAS526555. FUSE is operated for NASA by the Johns Hopkins University under contract NAS5-32985. I thank S. Redfield, G. Harper, and A. Brown for their help with aspects of this investigation. 


\section{References}

Ayres, T.R., Linsky J.L. 1980, ApJ 241, 279

Ayres, T.R., Simon, T., Stern, R., Drake, S., Wood, B., Brown, A. 1998, ApJ 496, 428 Ayres, T.R., et al. 2003, ApJ 583, 963

Bopp, B.W., Stencel, R.E. 1981, ApJ 247, L131

Moos, W.H., et al. 2000, ApJ 538, L1

Redfield, S., et al. 2003, ApJ 585, 993

Simon, T., Drake, S.A. 1989, ApJ 346, 303

Stauffer, J.R. 1991, in: S. Catalano \& J.R. Stauffer (eds.), Angular Momentum Evolution of Young Stars (Dordrecht: Kluwer), 117

Vaiana, G.S., Rosner, R. 1978, ARA\&A 16, 393

Wood, B.E., Linsky, J.L., Ayres, T.R. 1997, ApJ 478, 745

Woodgate, B.E., et al. 1998, PASP 110, 1183 